\title{
The Distribution of M2 Macrophage and Treg in Nasopharyngeal Carcinoma Tumor Tissue and the Correlation with TNM Status and Clinical Stage
}

\author{
Siti Hamidatul Aliyah ${ }^{1,2}$, Yustina Nuke Ardiyan ${ }^{3}$, Iffah Mardhiyah ${ }^{4}$, Camelia \\ Herdini $^{5}$, Ery Kus Dwianingsih ${ }^{6}$, Sumartiningsih Aning ${ }^{7}$, Niken Satuti Nur \\ Handayani $^{1}$, Widya Asmara ${ }^{8}$, Jajah Fachiroh ${ }^{9}$, Dewi Kartikawati Paramita ${ }^{7,9 *}$
}

\begin{abstract}
Objective: This study aimed to identify the distribution of M2 macrophage and Treg in Nasopharyngeal Carcinoma (NPC) tumor tissue samples. The presence of these two groups of cells was further correlated to clinical stage, tumor size, the lymphatic node involvement, and metastasis. Methods: The total of 50 formalin-fixed paraffin-embedded (FFPE) NPC tissue samples was collected retrospectively ( 27 samples) and prospectively ( 23 samples). Samples were FFPE tissue slices. Immunohistochemistry was done on the FFPE tissue slides using anti-CD-163 and anti-FoxP-3 antibodies for M2 macrophage and Treg detection, respectively. The M2 macrophage interpretation was performed by eye-balling method and the score was divided into 0 (negative), 1 (scant), 2 (focal), and 3 (abundant). The average number of Treg FOXP3+ cells in 5 high power fields (HPF) was calculated. The relationship of M2 macrophage and Treg was tested with Spearman's correlation. The relationship between M2 macrophage and Treg with clinical stage, tumor size, node involvement and metastasis was tested by chi square, with $p<0.1$. Results: M2 macrophage and Treg were positive correlated ( $\mathrm{r}=0.469, \mathrm{p}<0.001)$. The presence of M2 macrophage and regulatory T cell (Treg) was significantly correlated to tumor size ( $\mathrm{p}=0.091$ for M2 macrophage and $\mathrm{p}=0.022$ for Treg) and clinical stage $(\mathrm{p}=0.030$ for M2 macrophage and $\mathrm{p}=0.002$ for Treg), but did not correlate with lymphatic node involvement and metastasis. Conclusions: In Epstein-Barr virus related NPC tumor microenvironment, the presence of M2 macrophage was correlated with Treg, and both types of the cells were correlated with tumor size and clinical stages.
\end{abstract}

Keywords: CD163- FoxP3- M2 Macrophage- Treg- Nasopharyngeal Carcinoma- Epstein-Barr virus

Asian Pac J Cancer Prev, 22 (11), 3447-3453

\section{Introduction}

Nasopharyngeal carcinoma (NPC) is a type of head and neck cancer in nasopharyngeal epithelial tissue. The incidence of NPC worldwide is rare, but endemic in several regions, such as Southeast Asia, China, Northeast India, North Africa and in the Inuit population (Eskimos) in Canada and Alaska (Parkin et al., 2005). In Indonesia with a population of around 266 Million, NPC is estimated to be ranked as fifth of all malignancies, with an incidence rate of 6.6 per 100,000 population (Bray et al., 2018). The development of NPC in endemic areas is closely related to Epstein-Barr virus (EBV) infection and chronic inflammation. Tumor cells in NPC interact with surrounding cells to form a microenvironment that supports tumor development.

Epstein-Barr Virus encoded RNAs (EBERs) is the most commonly found viral transcript in cells of latently infected with EBV, including NPC cells, and the encoded gene is transcribed by RNA polymerase III (Takada, 2012). EBERs can induce expression of proinflammatory cytokines in NPC cells, which is dependent on retinoic acid-inducible gene I (RIG1) and toll-like receptor 3 (TLR3) activation (Duan et al., 2015; Li et al., 2015).

${ }^{1}$ Postgraduate Program, Faculty of Biology, Universitas Gadjah Mada, Yogyakarta, Indonesia. ${ }^{2}$ Pharmacy Program, Sekolah Tinggi Ilmu Kesehatan Harapan Ibu, Jambi, Indonesia. ${ }^{3}$ Department of Histology, Faculty of Medicine, Duta Wacana Christian University, Yogyakarta. ${ }^{4}$ Faculty of Dentistry, Universitas Gadjah Mada, Yogyakarta, Indonesia. ${ }^{5}$ Department of Otorhinolaryngology Head and Neck Surgery, Faculty of Medicine, Public Health and Nursing, Universitas Gadjah Mada, Yogyakarta, Indonesia. ${ }^{6}$ Department of Anatomical Pathology, Faculty of Medicine, Universitas Gadjah Mada, Yogyakarta, Indonesia. ${ }^{7}$ Integrated Research Laboratory, Faculty of Medicine, Public Health and Nursing, Universitas Gadjah Mada, Yogyakarta, Indonesia. ${ }^{8}$ Department of Microbiology, Faculty of Veterinary Medicine, Universitas Gadjah Mada, Yogyakarta, Indonesia. ${ }^{9}$ Department of Histology and Cell Biology, Faculty of Medicine, Public Health and Nursing, Universitas Gadjah Mada, Yogyakarta, Indonesia. *For Correspondence: dkparamita@ugm.ac.id. Siti Hamidatul 'Aliyah and Yustina Nuke Ardiyan have equal contribution in this study. 
Proinflammatory cytokines resulting from the interaction of EBERs and TLR-3 attract M2 macrophage that leads in Treg recruitment (Li et al., 2015). Proinflammatory cytokines can modulate polarization of macrophages primarily into M2 macrophage. The density of M2 macrophages in the tumor microenvironment has been found as a poor prognostic marker for various carcinomas (Cho et al., 2012). It is thought that M2 macrophages promote tumor growth by suppressing the immune system (Ooft et al., 2018; Wang et al., 2017). M2 macrophages have been shown to play a key role in immune suppression (Chen et al., 2012). M2 macrophage can recruit mature Treg by secreting cytokines such as CCL22 and CCL18, and M2 macrophages can modulate the conversion of naïve $T$ cells into Treg by secreting transforming growth factor-beta (TGF- $\beta$ ) and Interluekin-10 (IL-10) (Mantovani et al., 2002). Tregs are directly involved in promoting angiogenesis in the tumor microenvironment, and also promote cancer through immune escape (Facciabene et al., 2012). Research on the relationship of M2 macrophage and Treg with prognosis and severity of several cancers has been widely studied. However, research of these two cells in the microenvironment of NPC is still limited; therefore, it needs to be studied further. This study aimed to identify the distribution of M2 macrophage and Treg in the cancerous tissue samples of NPC. The presence of these two groups of cells was further correlated to staging, tumor size, the lymphatic node involvement, and metastasis.

\section{Materials and Methods}

This research was a descriptive observational study with a cross-sectional approach. Sampling technique using consecutive sampling with a minimum sample size for M2 Macrophage was 27 samples and Treg was 21 samples with the proportion of M2 macrophage (65\%) or Treg (77\%) in NPC (Ooft et al., 2017) .

\section{Study subjects}

The total of 50 formalin-fixed paraffin embedded (FFPE) samples were collected retrospectively (27 samples) and prospectively (23 samples) from the Department of Pathological Anatomy, Dr. Sardjito General Hospital, Yogyakarta, Indonesia. All of the samples were derived from patients in Dr. Sardjito General Hospital, Yogyakarta, Indonesia, at their first visit prior to any therapeutic treatment. The retrospective samples were collected from $2008-2010$ and the prospective samples were collected during April 2019 to February 2020. This study was approved by The Medical and Health Research Ethics Committee of the Faculty of Medicine, Public Health and Nursing, Universitas Gadjah Mada, Yogyakarta, Indonesia (No. KE/FK/0050/EC/2019).

\section{Immunohistochemistry (IHC) staining}

The FFPE samples were sliced at $3 \mu \mathrm{m}$ thick. Immunohistochemistry staining was performed by using Mouse and Rabbit Specific HRP/DAB IHC Detection Kit-Micro-polymer (Cat No: ab236466, Abcam, Cambridge, UK). Antigen retrieval was performed by using a Tris EDTA pH 9,0 for M2 macrophage and citrate buffer PH 6,0 for Treg. Monoclonal anti-CD163 antibody (clone EPR19518, Abcam, Cambridge, UK) at 1:500 dilution in phosphate buffer saline (PBS) used to detect M2 macrophages and monoclonal anti-FoxP3 antibody (clone 236A/E7, Abcam, Cambridge, UK) at 1:100 dilution in PBS used to detect Treg. Visualization was performed by using diamine-benzidine (DAB) (Abcam, Cambridge, UK) at 1:50 dilution in DAB substrate according to the manufacturer's instructions. Liver mouse tissue was used for M2 macrophage positive control and human tonsil for Treg positive control.

\section{Immunohistochemistry (IHC) assessment}

For CD163 in M2 macrophages, each section was screened at low-power magnification to identify the areas with the highest staining density. The positive expression of CD163 was defined by a granular cytoplasm or a cytoplasmic and membrane staining pattern. The expression CD163 was classified into four grades (0-3): negative (0), no staining; scant (1), a small amount of scattered staining; focal (2), concentrated staining with an irregular and non-continuous focus; and abundant and (3), concentrated stains with an extensive and continuous focus. This classification system was similar to the system used in a previous study (Fujii et al., 2012) as seen in Figure 2. Based on the result of the expression levels of CD163, they were classified into the "high" group for scores of 2-3 and the "low" group for scores of $0-1$ (Yu et al., 2018). The expression of Foxp3 showed the staining in the nucleus of T cells. Five high-power fields (HPF) were randomly selected. The number of positive cells were counted in each HPF, and the expression data identified as the mean of the number of positive cells in $5 \mathrm{HPF}$ (Wang et al., 2017). Each section was scored independently by two pathologists (Ardiyan et al., 2020). The average values from the two pathologists were used for further analysis.

\section{Statistical analysis}

The correlation between M2 macrophage and Treg were analyzed using Spearman correlation. The analysis of correlation between the M2 macrophage and Treg with TNM and clinical stage were analyzed using Chi square tests. All tests were performed using SPSS 18 (IBM Corp., Armonk, NY). All p-values presented were two-sided with $\mathrm{p} \leq 0.1$ defined as statistically significant.

\section{Results}

\section{Demographic and clinicopathological features of NPC patients}

Fifty (50) patients with relatively complete clinicopathological data extracted from medical records were included in this study. Seventy-four percent (74\%; $35 / 50)$ were male patients and $26 \%(13 / 50)$ were female patients with a median age of 51 years (range, 18-79 years). Forty-six percent $(46 \% ; 23 / 50)$ of the patients had T1- T2 tumor size, and 54\% (27/50) had T3-T4 tumor size. Ten percent $(10 \% ; 5 / 50)$ had negative node involvement (N0) and 90\% (45/50) were N1-3. Ninety-six percent $(96 \% ; 48 / 50)$ of the patients suffered from locoregional 
Table 1. Demographic and Clinicopathologic Data of NPC Patients

\begin{tabular}{lcc}
\hline Demographic & Patient $(\mathrm{n}=50)$ & Patient $(\%)$ \\
\hline Gender (n) & 37 & 74 \\
Male & 13 & 26 \\
Female & & \\
Age (yr) & 11 & 22 \\
18-40 & 30 & 60 \\
$41-60$ & 9 & 18 \\
$>60$ & & \\
T status & 23 & 46 \\
T1-T2 & 27 & 54 \\
T3-T4 & & \\
N status & 5 & 10 \\
N0 & 45 & 90 \\
N1-N2-N3 & & \\
M status & & \\
M0 & 48 & 96 \\
M1 & 2 & 46 \\
Stage & & 46 \\
II & 4 & \\
III & 23 & \\
IV & & \\
\hline
\end{tabular}
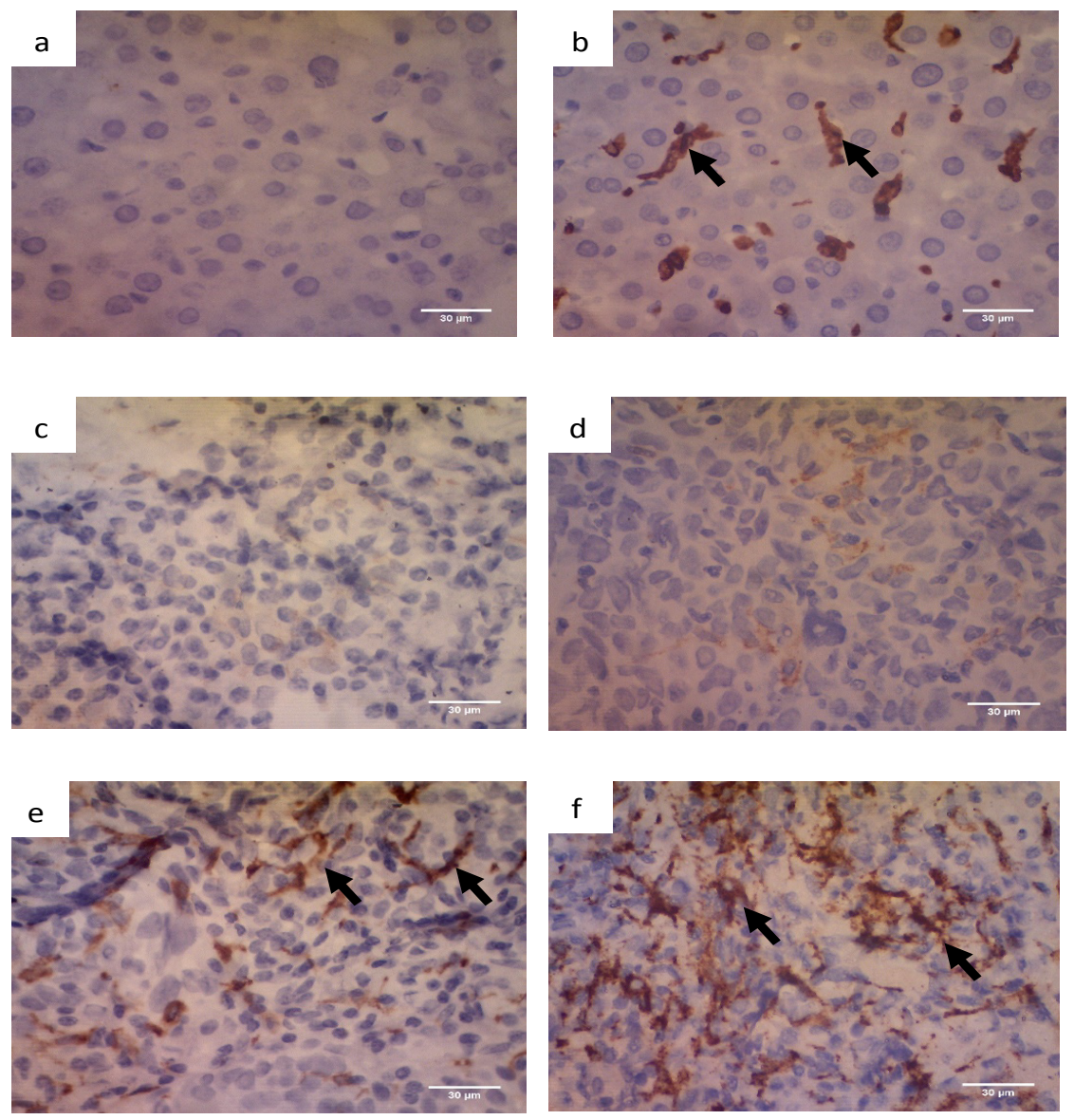

Figure 1. Immunohistochemistry of M2 Macrophage Using Anti-CD163 Antibody. Positive staining was shown on the membrane and cytoplasm (black arrow). The images were at 400x magnification. a. negative control (liver mouse) without anti-CD163 antibody, b. positive control (liver mouse) with anti-CD163 antibody, c. negative (0 score), no staining; d. scant (1 score), a small amount of scattered staining; e. focal (2 score), concentrated stains with an irregular and non-continuous focus; and f. abundant (3 score). Scale bar, $30 \mu \mathrm{m}$. 
Table 2. The Correlation among the Clinical Parameters, M2 Macrophage and Tregs (n=50)

\begin{tabular}{|c|c|c|c|c|c|c|}
\hline \multirow[t]{2}{*}{ Clinical Parameters } & \multicolumn{3}{|c|}{ M2 Macrophage } & \multicolumn{3}{|c|}{ Treg } \\
\hline & Low $(n=17)$ & $\operatorname{High}(n=33)$ & p-value & $\leq \operatorname{median}(n=24)$ & $>$ median $(n=26)$ & $\mathrm{p}$-value \\
\hline \multicolumn{7}{|l|}{ T stage } \\
\hline $\mathrm{T} 1-\mathrm{T} 2$ & 5 & 18 & $0.091 *$ & 7 & 16 & $0.022 *$ \\
\hline T3-T4 & 12 & 15 & & 17 & 10 & \\
\hline \multicolumn{7}{|l|}{$\mathrm{N}$ stage } \\
\hline N0 & 2 & 3 & 0.765 & 1 & 4 & 0.187 \\
\hline N1-N3 & 15 & 30 & & 23 & 22 & \\
\hline \multicolumn{7}{|l|}{ M stage } \\
\hline M0 & 17 & 31 & 0.3 & 22 & 24 & 0.149 \\
\hline M1 & 0 & 2 & & 2 & 0 & \\
\hline \multicolumn{7}{|l|}{ Clinical Stage } \\
\hline II & 0 & 4 & $0.030^{*}$ & 0 & 4 & $0.002 *$ \\
\hline III & 5 & 18 & & 7 & 16 & \\
\hline IV & 12 & 11 & & 17 & 6 & \\
\hline
\end{tabular}

Correlation between M2 macrophage and Treg; and correlation between M2 macrophage, Treg with TNM and Clinical stages

Spearman's correlation revealed a positive correlation between M2 macrophages and Treg in NPC tissue $(r=0.469$, $\mathrm{p}=0.001)$. The distributions of the characteristics of the sample sets within the scoring groups are summarized in Table 2. The presence of M2 macrophages and Treg was significantly correlated with $\mathrm{T}$ (tumor size) $(\mathrm{p}=0.091$ for M2 and $\mathrm{p}=0.022$ for Treg). The presence of M2 macrophages and Treg was also significantly correlated with clinical stage $(p=0.030$ for $M 2$ and $p=0.002$ for Treg) (Table 2).

\section{Discussion}

Nasopharyngeal carcinoma (NPC) is a common cancer of the human head and neck which is associated with genetic predisposition, Epstein-Barr virus (EBV) infection, and environmental factors. Global cancer statistic 2018 report found 129,079 cases of nasopharyngeal cancer worldwide, and 93,416 (72.37\%) were in males and $35,663(27.63 \%)$ were in females with a ratio of $2.62: 1$. In Southeast Asia, from a a total of 34,681 new cases, there were 25,895 cases $(74.67 \%$ ) diagnosed in males and 8,786 cases $(25.33 \%)$ in females, with a ratio of 2.95:1 (Bray et al., 2018; Duc and Ai, 2020). Data in the Indonesian Cancer Registry reported that NPC is more common in males, and is the second most common type of cancer in men after lung cancer. From the 50 patients included in this study, $74 \%(37 / 50)$ patients were male and $26 \%$ $(13 / 50)$ were female. Twenty-two percent $(22 \% ; 11 / 50)$ of the patients were aged between $18-40$ years, $60 \%(30 / 50)$ were 41-60 years old and 18\% (9/50) were over 60 years old. The number of patients increased after the age of 30 years, and reached a peak between 50 and 59 years. Our

\section{M2 Macrophage Density in NPC Patient}

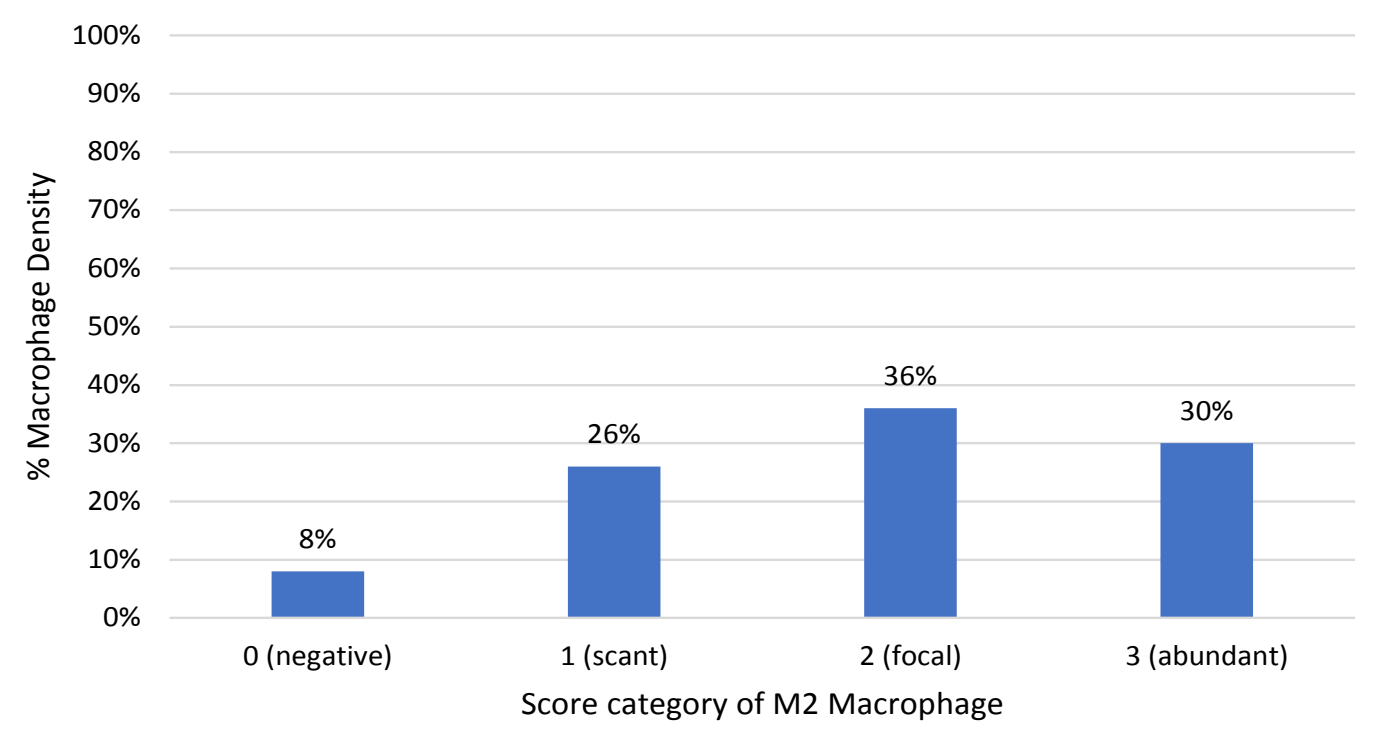

Figure 2. M2 Macrophage (CD163) Density in Patients with NPC. Eight percent $(8 \% ; 4 / 50)$ of patients were negative result of CD $163,26 \%(13 / 50)$ were scant grade, $36 \%(18 / 50)$ were focal grade and $30 \%(15 / 50)$ were abundant grade. 

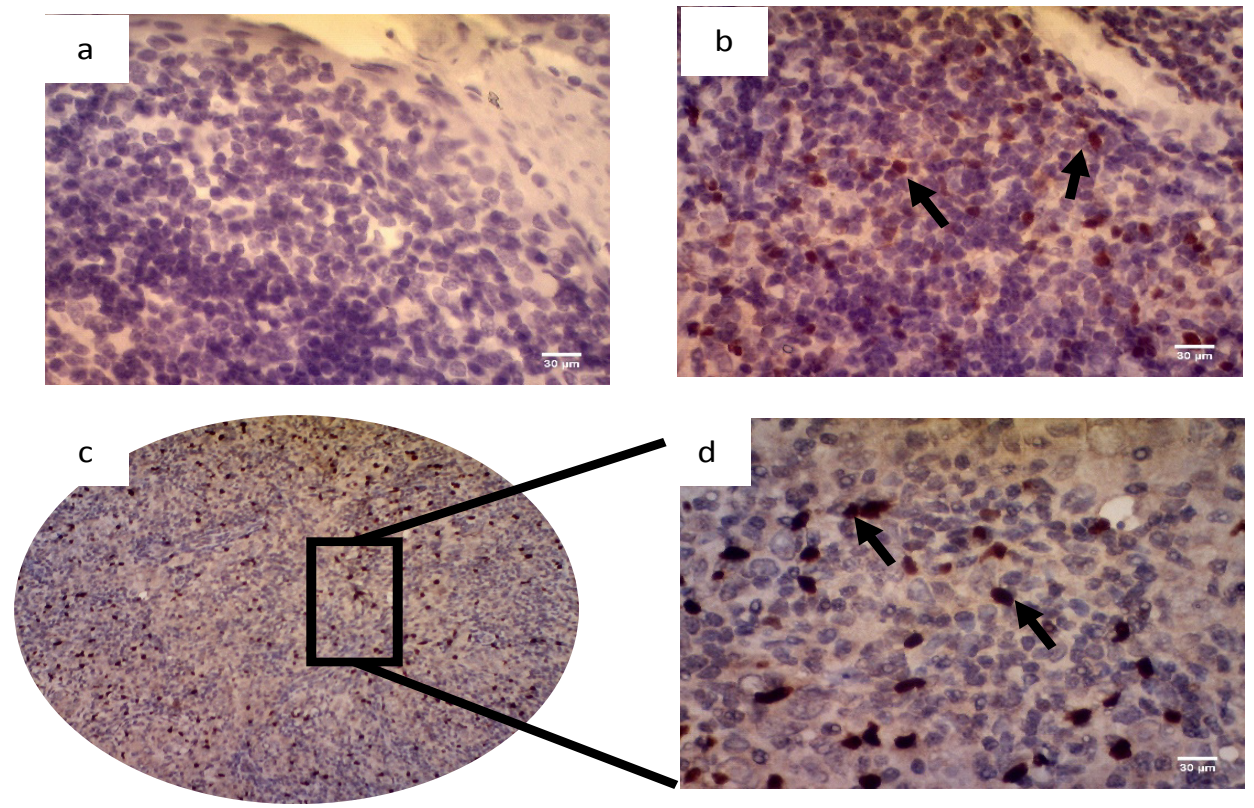

Figure 3. Immunohistochemistry of Treg Using Anti-FoxP3 Antibody. Positive staining was shown in the nucleus (black arrow). The images were taken with a 400x magnification. a. negative control (human tonsil) without antiFoxP3 antibody, b. positive control (human tonsil) with anti-FoxP3 antibody, c. positive result in NPC tissue (100x magnification), and d. positive result in NPC tissue (400x magnification). Scale bar, $30 \mu \mathrm{m}$.

study results were similar to studies in high-incidence areas in China (Parkin et al., 2005; Bray et al , 2018).

The most common NPC subtype in endemic areas including Indonesia is the undifferentiated carcinoma subtype, which is $100 \%$ related to EBV infection. The involvement of EBV infection in NPC cells causes an interaction between EBV and host cells. The interaction between EBV RNAs (EBERs) and TLR3 on host cells leads to the increase of inflammatory cytokine expression, including tumor necrosis factor-alpha (TNF $\alpha$ ), IL-6 and IL-1 $\alpha$. Those cytokines potentially recruit and activate macrophages, leading to a pro-tumorigenic microenvironment for solid tumor growth (Li et al., 2015).

Macrophages in the tumor microenvironment serve as tumor facilitators for cell migration and invasion, extracellular matrix degradation, and angiogenesis. Macrophages may be differentiated into two types: type 1 (M1 macrophage), which is activated classically and the alternative type 2 (M2 macrophages) (Mantovani et al., 2002). M1 macrophage is considered as proinflammatory and antitumoral, while M2 macrophages encourage tumor development, possessing immunosuppressive and protumoral effects (Galdiero et al., 2013; Ruffellet al., 2012). M2 macrophage can recruit mature Treg and modulate the conversion of naive T cells to Treg (Mantovani et al., 2002). Tregs play a role in immunosuppression and immune escape in tumor microenvironment (Chen et al., 2019). Research on M2 macrophage and Treg in EBV

\section{Number of Treg in NPC Tissue}

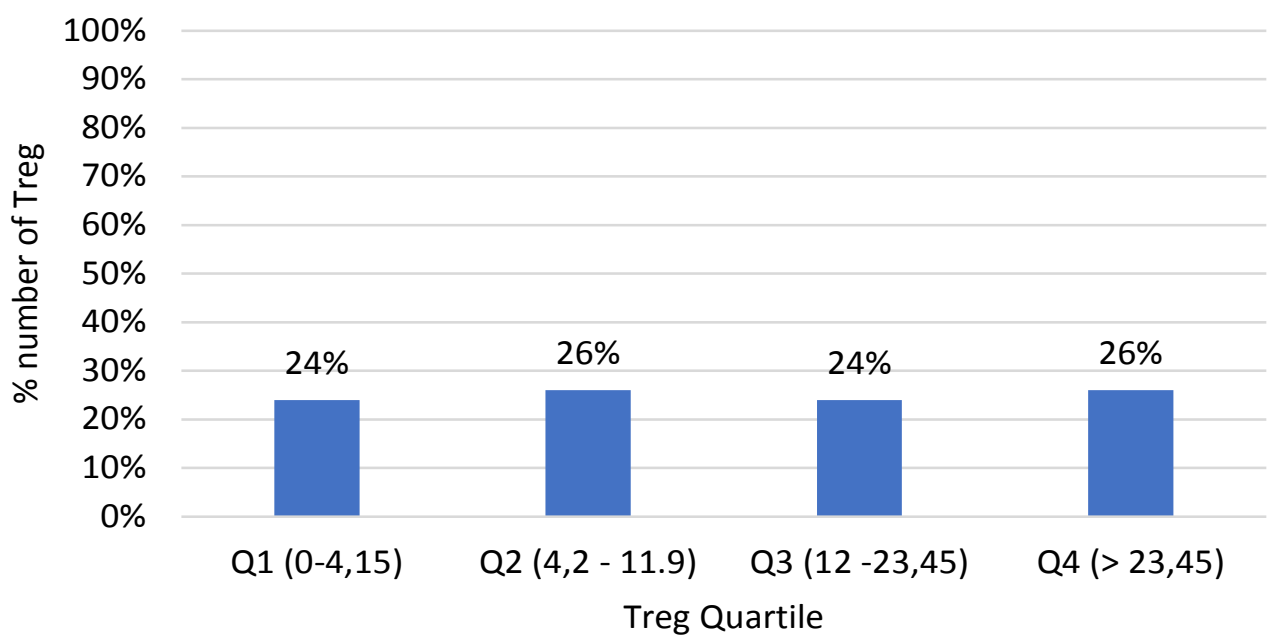

Figure 4. Quartile Group of Tregs. The results were grouped into four categories: Quartile $1(0-4.15)$, Quartile 2 (4.2-11.9), Quartile $3(12-23.45)$, and Quartile $4(>23.45)$. Twenty-four percent $(24 \% ; 12 / 50)$ of patients were in Q1, $26 \%(13 / 50)$ were in Q2, 24\% (12/50) were in Q3 and 26\% (12/50) were in Q4. 
related NPC has not yet been described. Several studies reported that M2 macrophage and Treg are closely related with worse survival and poor prognosis in several types of cancer. M2 macrophage and Treg can be important indicator factors to determine therapeutic targets (Ooft et al., 2018; Wang et al., 2017).

The results of this study indicated that M2 macrophage and Treg have a positive correlation while the amounts of $46.9 \%$, and $53.1 \%$ of the correlation were affected by other factors, possibly by the EBV oncogene Latent membrane Protein 2 (LMP1) and Epstein-Barr Nuclear Antigen (EBNA1). Wang et al., (2017) reported that NPC cell line culture (CNE1, CNE2, and 5-8F) can recruit M2 macrophage and furthermore, Treg was recruited by M2 macrophage. Their study also showed that M2 macrophage have a positive association with Treg cells. Macrophage secretes chemokines such as CCL22, CCL17, and CCL18 to recruit Treg and modulate the immune response during the tumorigenic process (Curiel et al., 2004; Mantovani, 2008). Another factor that can recruit Treg in tumor area is LMP1. LMP1 can recruit Tregs through the activation of NF-kB signaling that will lead the upregulation of the CCL20 chemokine (Baumforth et al., 2008). High CCL20 expression increases the migration of Tregs cells towards the tumor (Baumforth et al., 2008; Tsao et al., 2014). Furthermore, LMP1 can also induce Tregs to secret IL-10 (Marshall et al., 2003) that plays a role in immune suppression. Besides M2 macrophage and LMP1, Wang et al., (2020) also report that EBNA1 induces the accumulation of Tregs through the conversion of naïve $\mathrm{T}$ cell into Tregs via upregulated TGF- $\beta 1$ and CCL 20 which will induce EBV-directed migration, and polarized-M2 macrophage to convert naïve T cells into Tregs.

Previous study showed that CD163 is a highly specific marker of M2 macrophages and has been studied in several aggressive tumors. The increased expression of CD163 was significantly associated with a poor prognosis in various cancers (Clear et al., 2010; He et al., 2014; Kridel et al., 2015; Tiainen et al., 2015). Yu et al., (2018) also reported that higher expression of CD163 predicted worse survival of NPC patients. This is probably due to M2 macrophages promoting tumor growth. Tumor associated macrophages (TAMs) that are dominated by M2 are known to induce Tregs recruitment and differentiation in the tumor microenvironment (Daurkin et al., 2011; Denning et al., 2007). M2 macrophage and Treg form a special niche, which promotes tumor progression (Kitamura et al., 2015). In our study, M2 macrophage and Treg were significant correlated with tumor size (T status) and clinical stages, but no correlation was found with the involvement of the lymphatic nodes nor with distant metastasis of NPC.

This study had several limitations, including the small number of the samples which may influence the results. This study only focused on the presence of M2 and Treg and their association with severity. Several parameters, including other cells in NPC tumor microenvironment (inflammatory cells, cancer associated fibroblasts, and endothelial cells), and EBV or cellular proteins that play a role in NPC growth were not included in this study. Since most of the samples (92\%) were at the advanced stage
NPC (III and IV), it is difficult to determine the correlation between the presence of M2 macrophage and Treg with TNM status and clinical stage objectively.

In conclusion, the presence of M2 macrophage significantly correlates with the presence of Treg in EBVrelated NPC tumor microenvironment. M2 macrophage and Treg were significantly correlated with tumor size and clinical stage. For further study, it is necessary to look at other parameters in the tumor microenvironment that play a role in tumor progression, and also assess the survival rate and other clinicopathology features as prognostic factors.

\section{Author Contribution Statement}

SHA, YNA and DKP were involved in planning. SHA, IM, NSNH drafted the manuscript and designed the figures. $\mathrm{CH}$ collected samples from the patients with NPC. EKD analyzed the IHC results. SHA and JF performed the statistical analysis. SHA, WA and DKP wrote the discussion and conclusions. After the first draft, all of the author discussed and commented on the manuscript. All authors read and approved the final version of the manuscript. SHA and DKP finalized the manuscript to be ready for submission.

\section{Acknowledgments}

We would like to express our gratitude to Histology Laboratory, Department of Histology and Cell Biology for the facilities during experiments; and Pathological Anatomy Laboratory, Department of Pathological Anatomy for providing the FFPE slides. Thank you to Ms. Sumaryati in Histology Laboratory for technical assistance.

\section{Funding statement}

This work was supported by the grant from Society Funding (Dana Masyarakat/DAMAS) Faculty of Medicine, Public Health and Nursing Universitas Gadjah Mada 2019 and Doctorate Dissertation Research (Penelitian Disertasi Doktor/PDD) 2020 No. 2071/ UN1/ DITLIT/DIT-LIT/PT/2020, Ministry of Education \& Culture Republic of Indonesia.

\section{Data statement}

Some data of this study were used for completing the thesis of Yustina Nuke Ardiyan and will be used in the doctoral study of Siti Hamidatul 'Aliyah.

\section{Conflict of interest}

The authors declare no potential conflict of interest.

\section{References}

Ardiyan YN, Paramita DK, Fachiroh J. (2020). The correlation of macrophagocytus M2 and T regulatory cell) with TNM Status and Clinical Stage (Unpublished master's thesis). Universitas Gadjah Mada, Yogyakarta.

Baumforth KRN, Birgersdotter A, Reynolds GM, et al (2008). Expression of the Epstein-Barr virus-encoded Epstein- 
Barr virus nuclear antigen 1 in Hodgkin's lymphoma cells mediates up-regulation of CCL20 and the migration of regulatory T cells. Am J Pathol, 173, 195-204.

Bray F, Ferlay J, Soerjomataram I, et al (2018). Global cancer statistics 2018: GLOBOCAN estimates of incidence and mortality worldwide for 36 cancers in 185 countries. $C A$ Cancer J Clin, 68, 394-424.

Chen J, Li G, Meng H, et al (2012). Upregulation of B7-H1 expression is associated with macrophage infiltration in hepatocellular carcinomas. Cancer Immunol Immunother, 61, 101-8.

Chen Y, Song Y, Du W, et al (2019). Tumor-associated macrophages: an accomplice in solid tumor progression. J Biomed Sci, 26, 1-13.

Cho HJ, Jung JI, Lim DY, et al (2012). Bone marrow-derived, alternatively activated macrophages enhance solid tumor growth and lung metastasis of mammary carcinoma cells in a Balb/C mouse orthotopic model. Breast Cancer Res, 14, R81 1-12.

Clear AJ, Lee AM, Calaminici M, et al (2010). Increased angiogenic sprouting in poor prognosis FL is associated with elevated numbers of CD163+ macrophages within the immediate sprouting microenvironment. Blood, 115, 5053-56.

Curiel TJ, Coukos G, Zou L, et al (2004). Specific recruitment of regulatory $\mathrm{T}$ cells in ovarian carcinoma fosters immune privilege and predicts reduced survival. Nat Med, 10, 942-49.

Daurkin I, Eruslanov E, Stoffs T, et al (2011). Tumor-associated macrophages mediate immunosuppression in the renal cancer microenvironment by activating the 15-lipoxygenase- 2 pathway. Cancer Res, 71, 6400-9.

Denning TL, Wang YC, Patel SR, et al (2007). Lamina propria macrophages and dendritic cells differentially induce regulatory and interleukin 17-producing $\mathrm{T}$ cell responses. Nat Immunol, 8, 1086-94.

Duan Y, Li Z, Cheng S, et al (2015). Nasopharyngeal carcinoma progression is mediated by EBER-triggered inflammation via the RIG-I pathway. Cancer Lett, 361, 67-74.

Duc Lao T, Ai Huyen Le T (2020). Epidemiology, incidence, mortality of cervical cancer in Southeast Asia and their relationship: an update report. Asian J Pharm Res Heal Care, 12, 97-101.

Facciabene A, Motz GT, Coukos G (2012). T-Regulatory cells: key players in tumor immune escape and angiogenesis. Cancer Res, 72, 2162-71.

Fujii N, Shomori K, Shiomi T, et al (2012). Cancer-associated fibroblasts and CD163-positive macrophages in oral squamous cell carcinoma: their clinicopathological and prognostic significance. J Oral Pathol Med, 41, 444-51.

Galdiero MR, Garlanda C, Jaillon S, et al (2013). Tumor associated macrophages and neutrophils in tumor progression. $J$ Cell Physiol, 228, 1404-12.

He KF, Zhang L, Huang CF, et al (2014). CD163+ tumorassociated macrophages correlated with poor prognosis and cancer stem cells in oral squamous cell carcinoma. Biomed Res Int, 2014, 838632

Kitamura T, Qian BZ, Soong D, et al (2015). CCL2-induced chemokine cascade promotes breast cancer metastasis by enhancing retention of metastasis-associated macrophages. $J$ Exp Med, 212, 1043-59.

Kridel R, Xerri L, Gelas-Dore B, et al (2015). The prognostic impact of CD163-positive macrophages in follicular lymphoma: a study from the $\mathrm{BC}$ cancer agency and the lymphoma study association. Clin Cancer Res, 21, 3428-35.

Li Z, Duan Y, Cheng S, et al (2015). EBV-encoded RNA via TLR3 induces inflammation in nasopharyngeal carcinoma. Oncotarget, 6, 24291-303.
Distribution of M2 Macrophage and Treg in NPC Tumor Tissue

Mantovani A (2008). From phagocyte diversity and activation to probiotics: back to Metchnikoff. Eur J Immunol, 38, 3269-73.

Mantovani A, Sozzani S, Locati M, et al (2002). Macrophage polarization: tumor-associated macrophages as a paradigm for polarized M2 mononuclear phagocytes. Trends Immunol, 23, 549-55.

Marshall NA, Vickers MA, Barker RN (2003). Regulatory T cells secreting IL-10 dominate the immune response to EBV latent membrane protein 1. J Immunol, 170, 6183-9.

Ooft ML, van Ipenburg JA, Braunius WW, et al (2017). Prognostic role of tumor infiltrating lymphocytes in EBV positive and EBV negative nasopharyngeal carcinoma. Oral Oncol, 71, 16-25.

Ooft ML, van Ipenburg JA, Sanders ME, et al (2018). Prognostic role of tumour-associated macrophages and regulatory $\mathrm{T}$ cells in EBV-positive and EBV-negative nasopharyngeal carcinoma. J Clin Pathol, 71, 267-274.

Parkin DM, Bray F, Ferlay J, et al (2005). Global cancer statistics, 2002. CA Cancer J Clin, 55, 74-108.

Ruffell B, Affara NI, Coussens LM (2012). Differential macrophage programming in the tumor microenvironment. Trends Immunol, 33, 119-26.

Takada K (2012). Role of EBER and BARF1 in nasopharyngeal carcinoma (NPC) tumorigenesis. Semin Cancer Biol, 22, 162-5.

Tiainen S, Tumelius R, Rilla K, et al (2015). High numbers of macrophages, especially M2-like (CD163-positive), correlate with hyaluronan accumulation and poor outcome in breast cancer. Histopathology, 66, 873-83.

Tsao SW, Yip YL, Tsang CM, et al (2014). Etiological factors of nasopharyngeal carcinoma. Oral Oncol, 50, 330-8.

Wang J, Huang H, Lu J, et al (2017). Tumor cells induced-M2 macrophage favors accumulation of Treg in nasopharyngeal carcinoma. Int J Clin Exp Pathol, 10, 8389-8401.

Wang J, Luo Y, Bi P, et al (2020). Mechanisms of EpsteinBarr virus nuclear antigen 1 favor Tregs accumulation in nasopharyngeal carcinoma. Cancer Med, 9, 5598-5608.

Yu Y, Ke L, Lv X, et al (2018). The prognostic significance of carcinoma-associated fibroblasts and tumor-associated macrophages in nasopharyngeal carcinoma. Cancer Manag, Res, 10, 1935-46.

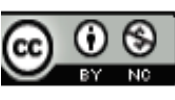

This work is licensed under a Creative Commons AttributionNon Commercial 4.0 International License. 